\title{
Evaluation of the accuracy of an offline seasonally-varying matrix transport model for simulating ideal age
}

\author{
Ann Bardin $^{a 1}$ and François Primeau ${ }^{a 2}$ \\ ${ }^{a}$ Department of Earth System Science, University of California, Irvine, California \\ ${ }^{1}$ abardin@uci.edu,corresponding author ${ }^{2}$ fprimeau@uci.edu \\ Keith Lindsay ${ }^{b}$ \\ ${ }^{b}$ Climate and Global Dynamics Division, National Center for Atmospheric Research, \\ Boulder, Colorado \\ klindsay@ucar.edu \\ Andrew Bradley ${ }^{c}$ \\ ${ }^{c}$ Center for Computing Research, Sandia National Laboratories, Albuquerque, New \\ Mexico \\ ambradl@sandia.gov
}

\begin{abstract}
Newton-Krylov solvers for ocean tracers have the potential to greatly decrease the computational costs of spinning up deep-ocean tracers, which can take several thousand model years to reach equilibrium with surface processes. One version of the algorithm uses offline tracer transport matrices to simulate an annual cycle of tracer concentrations and applies Newton's method to find concentrations that are periodic in time. Here we present the impact of time-averaging the transport matrices on the equilibrium values of an ideal-age tracer. We compared annually-averaged, monthly-averaged, and 5-day-averaged transport matrices to an online simulation using the ocean component of the Community Earth System Model (CESM) with a nominal
\end{abstract}


horizontal resolution of $1^{\circ} \times 1^{\circ}$ and 60 vertical levels. We found that increasing the time resolution of the offline transport model reduced a low age bias from $12 \%$ for the annually-averaged transport matrices, to $4 \%$ for the monthly-averaged transport matrices, and to less than $2 \%$ for the transport matrices constructed from 5-day averages. The largest differences were in areas with strong seasonal changes in the circulation, such as the Northern Indian Ocean. For many applications the relatively small bias obtained using the offline model makes the offline approach attractive because it uses significantly less computer resources and is simpler to set up and run.

Keywords: Implicit solver, ideal age, Newton-Krylov, offline, transport matrix, Global Ocean Modeling

1. Introduction

Tracers in the deep ocean take thousands of years to equilibrate with their 3 sources and sinks (e.g. Primeau and Deleersnijder, 2009). As a result, ocean 4 general circulation models that aim to simulate climatological tracers need 5 long spin-up runs before reaching their equilibrium state. Even for models of moderate spatial resolution the computational burden is significant. The problem is further compounded by the CFL stability conditions, which impose ever smaller time-step size as resolution increases. Consequently, the push for models of ever increasing resolution means that the required computational resources will continue to outpace the expected increases in computational resources for the foreseeable future.

To avoid the excessive computational burden associated with the long time to tracer equilibrium, Li and Primeau (2008) and also Khatiwala (2008) 
showed that a Newton-Krylov algorithm can be used to greatly decrease the computational time needed to spin up biogeochemical tracers to their seasonally-varying equilibrium state. The method solves for the periodic state of the annual cycle without solving for the transient spin-up of the tracers. This is accomplished by solving the coupled system of algebraic equations that propagate the tracer state of the ocean at the beginning of the year forward in time by exactly one year, such that the same tracer state is achieved at the end of the year. The algorithms proposed by Li and Primeau (2008) and also by Khatiwala (2008) used an offline tracer transport matrix with monthly temporal resolution to simulate the time-dependent seasonal cycle; however, the offline simulation is not an essential part of the algorithm. The Newton-Krylov algorithm can also be applied by using the full dynamical model to simulate the seasonal cycle needed to evaluate the transformation of the tracer (Lindsay, submitted). The tracer transport matrix is only indispensable for the construction of an effective preconditioner. In fact, using the online dynamical model to propagate the model tracer state forward for one year has the advantage of eliminating the errors incurred by approximating the continuous time-dependence of the advection-diffusion operator by a discrete set of 12 monthly transport operators. The main advantages of the offline tracer transport matrix are that it uses significantly fewer computational resources and is in general much simpler to set up and run. Other useful additional capabilities are the ease of applying filtering operations to the transport matrix operators; the ease of using subsets of the operators (such as advection only); and the ability to restrict simulations to specific regions. 
There is a substantial and growing body of work that is based on a transport matrix constructed by averaging the circulation of an OGCM (e.g. Primeau, 2005; Khatiwala et al., 2005; Kwon and Primeau, 2006; Kriest and Oschlies, 2011; Graven et al., 2012; Prieß et al., 2013; Bardin et al., 2014; Letscher et al., 2015), yet the error incurred by approximating the timedependence of the circulation by step-wise constant tracer-transport matrices has never been rigorously assessed. The goal of this study is to start to fill in this gap by comparing an equilibrium tracer field obtained with an online simulation to one obtained from an offline matrix-transport model in which the seasonal cycle is represented by (i) an annually averaged matrix, (ii) a set of 12 monthly averaged matrices, and (iii) a set of 73 5-day averaged matrices. The tracer we consider is the ideal age (e.g. England, 1995; Deleersnijder et al., 2001; Primeau, 2005), because it nicely illustrates the long timescales of ocean transport and because it is straightforward to implement without any complicated tracer source-sink functions.

The main objective here is to quantify the difference the averaging of advection and diffusion fields that occurs in the offline approach makes on the computed ideal age, compared to using the online model with the full time resolution of the parent dynamic model. Are the differences using the offline approach sufficiently small that the precomputed transport matrices can be used for biogeochemical sensitivity studies, for calibrating sub-gridscale eddy diffusivity parameterization, or for the spin-up of biogeochemical tracers for the purpose of initializing transient simulations? We lay the groundwork by which to answer these questions quantitatively. 


\section{Methods}

\subsection{Ideal Age}

The ideal age is defined as the mean time since a water parcel last had contact with the surface. It can be computed by solving the following initial value problem

$$
\left\{\begin{aligned}
\frac{\partial a}{\partial t}+\nabla \cdot[\mathbf{u}-\mathbf{K} \nabla] a & =1, \\
\widehat{\mathbf{n}} \cdot \mathbf{K} \nabla a & =0, \text { on all solid boundaries, } \\
a & =0 \text { at the sea surface, } a\left(t_{o}\right)=a_{o},
\end{aligned}\right.
$$

where $a$ is the age, $\mathbf{u}$ is the fluid velocity, and $\mathbf{K}$ is the eddy diffusion tensor. The bold notation denotes vectors or tensors. "1" on the right hand side of the equation is an age source or rate of aging, i.e. 1 year per year.

We consider the case where the ocean circulation is perfectly periodic with a period of $\Delta T=1$ year. This condition is satisfied provided the fluid velocity and eddy diffusivity tensor are both periodic with

$$
\begin{aligned}
\mathbf{u}(t+\Delta T) & =\mathbf{u}(t), \\
\mathbf{K}(t+\Delta T) & =\mathbf{K}(t) .
\end{aligned}
$$

We are interested in the long-time asymptotic solution to equation (1) at which point the transients have died out so that the ideal age is also periodic with $a(t+\Delta T)=a(t)$.

\subsection{The Dynamical Ocean Model}

For this study we simulate the ocean circulation using the dynamical ocean model component (POP2) of the Community Earth System Model 
(CESM), version 1.2 (Smith et al., 2010). The physical circulation was "spunup" from rest for 250 years using the Common Ocean-ice Reference Experiments (CORE) climatological normal year forcing (Large and Yeager, 2004; Griffies et al., 2009; Large and Yeager, 2009), in which the same seasonal cycle is repeated every model year. The temperature and salinity were initialized from the January-mean climatology. Following the spin-up of the dynamics, the dynamical ocean model was run for an additional year during which all quantities needed to construct the model's advection-diffusion transport operators in matrix form with monthly or 5-day time resolution were saved. The monthly-averaged or 5-day-averaged transport matrices provide representations of the seasonal cycle. These transport matrices can also be averaged to produce the annually averaged transport matrix. Details of the construction of the offline transport matrices from the parent dynamical model are given in Bardin et al. (2014).

The length of the dynamical model spin-up was chosen based on the tradeoff between the use of computational resources and wall-clock time, and the model simulation time needed for the transients in the momentum equations to decay. The 250-year duration of the spin-up is inadequate for the transients in $\mathbf{u}$ and $\mathbf{K}$ to completely die out, but we nevertheless enforce the periodicity of the circulation when we solve equation (1). By doing so we suppress the effect of any intrinsic inter-annual variability on the ideal age. For the online model this is done by reinitializing the dynamical-model to the same model-year state conditions at the beginning of each year of iteration as described in Lindsay (submitted). For the offline model this is done by recycling the same transport matrices as described in Bardin et al. (2014). 
Other details of the model configuration are as follows. The model has a dipolar grid with the convergence point normally found at the geographic North Pole displaced into Greenland; the transition from the grid following latitudinal and longitudinal lines starts at the Equator. The nominal $1^{\circ} \times 1^{\circ}$ horizontal grid has a higher meridional resolution of about $0.3^{\circ}$ approaching the equator. In the vertical dimension there are 60 depth levels with vertical resolution of $10 \mathrm{~m}$ for the top 16 layers, increasing to $250 \mathrm{~m}$ near the bottom. The thickness of the top-most layer is allowed to vary from its $10 \mathrm{~m}$ thickness to include regional and temporal variations in sea surface height. Details of the numerical methods and discretization are given in the POP Reference Manual (Smith et al., 2010).

Transport of tracers in the model is achieved by a combination of explicitly resolved currents and parameterized sub-gridscale transport processes. Tracer transport is divided into an advection term, a "horizontal diffusion" term, and a "vertical diffusion" term. The advection term includes the explicitly resolved velocity as well as a bolus velocity that arises from the Gent McWilliams (GM) parameterization of mesoscale eddies (Gent and McWilliams, 1990). The advection term also incorporates a sill-overflow parameterization for the deep channel overflows in the Denmark Strait and the Faroe Banks Channel; and for continental shelf overflows in the Weddell and Ross Seas (Briegleb et al., 2010; Danabasoglu et al., 2010). A third-order upwind advection scheme is used to compute the advective tendencies of the explicitly-resolved and bolus velocities. The model also includes a purely diffusive parameterization of mixing by mesoscale eddies in the form of a diffusion tensor that is rotated so that the large "horizontal" diffusion acts along 
isopycnal surfaces rather than across them (Redi, 1982). Thus the vertical diffusion term combines the vertical component of the along-isopycnal diffusion together with the diffusion from the K-profile parameterization (KPP) (Large et al., 1994), which accounts for the mixing induced by convective instabilities, shear instabilities, destabilizing tendencies associated with vertical gradients of salinity and temperature, as well as tidal mixing. The horizontal diffusion term captures the horizontal component of the along isopycnal diffusion.

\subsection{Newton Krylov Solver}

After being discretized in space the initial value problem (1) can be expressed as a system of ordinary differential equations, which we denote symbolically as follows

$$
\left\{\begin{aligned}
\frac{d}{d t} a(t) & =\mathbf{f}(t, a(t)) \\
a\left(t_{0}\right) & =a_{0},
\end{aligned}\right.
$$

and whose solution can be expressed in terms of the following integral

$$
a(t)=a\left(t_{0}\right)+\int_{t_{0}}^{t} \mathbf{f}\left(t^{\prime}, a\left(t^{\prime}\right)\right) d t^{\prime} .
$$

3 We define $G$ as the integral over the time period

$$
G\left(a\left(t_{0}\right)\right) \equiv \int_{t_{0}}^{t_{0}+\Delta T} \mathbf{f}\left(t^{\prime}, a\left(t^{\prime}\right)\right) d t^{\prime},
$$

such that the equilibrium condition, $a\left(t_{0}+\Delta T\right)=a\left(t_{0}\right)$, is then given by

$$
G\left(a\left(t_{0}\right)\right)=0 .
$$

Further discretizing the time variable converts equation (6) into a system of algebraic equations, which can be solved using a Newton-Krylov method. We 
note that evaluating $G$ is equivalent to running the ideal age model forward in time for one year $(\Delta T)$ then subtracting the initial tracer state $\left(a\left(t_{0}\right)\right)$ from the final tracer state $\left(a\left(t_{0}+\Delta T\right)\right)$.

We solve equation (6) using a Newton-Krylov algorithm in which we evaluate $G\left(a\left(t_{0}\right)\right)$ using the full online CESM-POP2 model (Lindsay, submitted) and using an offline model in which the advective-diffusive tracer flux divergence is computed using a discrete set of time-averaged tracer-transport matrices following Bardin et al. (2014).

Because the governing equation for the ideal age is linear, we can be assured that the solution is unique and that any difference we find between the online and offline solution can be attributed to the impact of using timeaveraged transport matrices in the offline model.

\subsection{Online Evaluation of $G$}

The online evaluation of $G$ consists of simulating the ideal age concurrently with the dynamical simulation. The temporal resolution with which the circulation evolves and the time discretization used to evaluate the integral defining $G$ are therefore the same as that used to time-step CESM-POP2. As previously mentioned, the periodicity of the flow is enforced by initializing the dynamical model to the same initial state (that is, the same temperature, salinity, velocity vectors, and sea-surface height) at the beginning of each one-year simulation (Lindsay, submitted). In CESM-POP2 the surface boundary condition for the ideal age tracer is implemented by resetting the ideal age to zero in the upper-most layer of the model at the end of every time-step. 


\subsection{Offline Evaluation of $G$}

For the offline evaluation of $G$ we use a discrete set of time-averaged transport matrices to compute the tracer flux divergence in the integrand of equation (5). Following Bardin et al. (2014), we separate the transport matrix into three terms

$$
\mathbf{T}=\mathbf{A}+\mathbf{D}+\mathbf{H}
$$

where $\mathbf{A}$ includes the effect of advection and the tracer-transport effects of the sill overflow parameterization, D provides the effect of vertical diffusion, and $\mathbf{H}$ provides the effect of horizontal diffusion. These operators correspond to the POP Reference Manual (Smith et al., 2010) advection and diffusion operators $\mathbf{A}=-\mathcal{L}_{T}, \mathbf{D}=\mathcal{D}_{V}$, and $\mathbf{H}=\mathcal{D}_{H}$. The transport matrices are sparse and square, with dimensions given by the number of ocean grid-cells in the parent model. $\mathbf{A}$ and $\mathbf{H}$ are generated from the dynamical model using an Impulse-Response Function (IRF) while running the dynamical model through a one-year cycle. At the same time, the vertical diffusion coefficient is recorded; this allows $\mathbf{D}$ to be generated by explicit coding (Bardin et al., 2014).

We evaluate the integral in (5) by time-stepping the offline tracer-transport model through the seasonal cycle, using a set of piece-wise constant tracertransport matrices. We consider three cases: a case with 73 5-day averaged matrices, a case with 12 monthly-averaged matrices, and a case with 1 annually-averaged matrix; with each case having a set of the three component matrices in (7). Within each period (5 days, one month, or one year) the equation is stepped forward in time using an implicit first-order backward-Euler scheme for the vertical diffusion; 3rd-order Adams-Bashforth 
for the advection; and first-order forward-Euler for horizontal diffusion and the age source-sink term. These schemes were chosen to accurately represent the methods of tracer transport implemented in the online model. The surface boundary condition implementation in CESM-POP2 was used when time-stepping the tracer-transport model. When using direct matrix inversion techniques, the surface boundary condition was implemented with a first-order loss term with a loss frequency $1 / \tau$ of $(1 \text { hour })^{-1}$. This value is sufficiently high to effectively clamp the surface value of the ideal age to zero.

\subsection{Convergence of the Newton-Krylov Solver}

To ensure that any differences between the online and offline solutions are due to the approximation made by using piece-wise constant tracer transport matrices in the offline model we must ensure that equation (6) is solved with sufficient accuracy. The criterion that we employ is that the residual drift in the ideal age be less than 0.01 years per year for $99 \%$ of the ocean volume. This threshold is analogous to the threshold used for the OCMIP-2 $\Delta{ }^{14} \mathrm{C}$ protocol (Lindsay, submitted).

Figure 1 shows a histogram of the remaining residuals after the convergence criterion is met for both the online and offline solutions. In a timedependent simulation the remaining residual is expected to decay exponentially with time. Consequently, the contribution to the error in the ideal age from the residual drift is expected to be small; certainly much smaller than the differences between the online and offline solutions presented in the following section. 

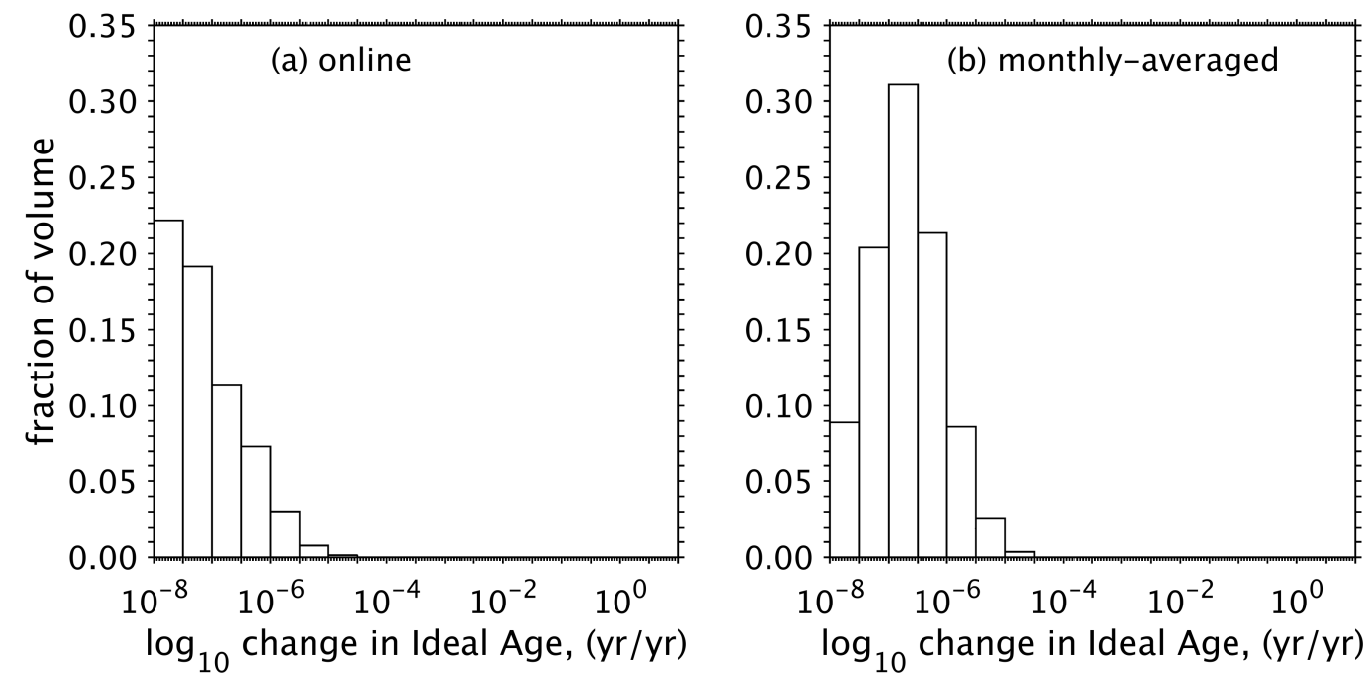

Figure 1: Histograms (a) and (b) show the distribution of the residual change over a oneyear simulation of the equilibrium solution of ideal age, (a) for an online simulation, and (b) for a simulation run offline using monthly-averaged transport operators. Note that the $\mathrm{x}$-axis is on a logarithmic scale. A typical criterion for acceptable convergence is that $99 \%$ of the volume has a residual of less than $10^{-2}$ years per year. The residual change is well below this criterion. 


\section{Comparison of Offline to Online Simulation Results}

In this section the results of the online simulation are compared with the results using annually-averaged, monthly-averaged, and 5-day-averaged transport matrices. The purpose of this exercise is to determine how much and where the biases are in the resulting equilibrium solution for ideal age, compared to an equilibrium solution based on running the dynamic model in an online simulation for the same year.

Figure 2 shows scatter-density diagrams comparing the ideal age equilibrium solutions obtained using the annually-averaged, monthly-averaged, and 5-day-averaged transport matrices to running online for the one-year simulation.

The top panel compares the annually-averaged case to the online case. The differences fall in a band below the one-to-one line on the scatter-density diagram, indicating that ignoring the seasonal cycle results in a too-young bias. On a volume-weighted basis, the ideal age computed using the annuallyaveraged transport operator produces ages that are $12 \%$ younger than the online simulation.

Using the monthly-averaged transport operator (middle panel) results in a significantly smaller bias than the annually-averaged operator. The density of points on the scatter density diagram is concentrated much more tightly along the one-to-one line. The volume-weighted bias is now only $-3.9 \%$.

The bottom panel in Figure 2 compares the equilibrium solution for the 5day-averaged transport operator to the online case. The tracer-tracer scatter density plot falls tightly on the one-to-one line with virtually no scatter. The volume-weighted bias is only $-1.6 \%$. 


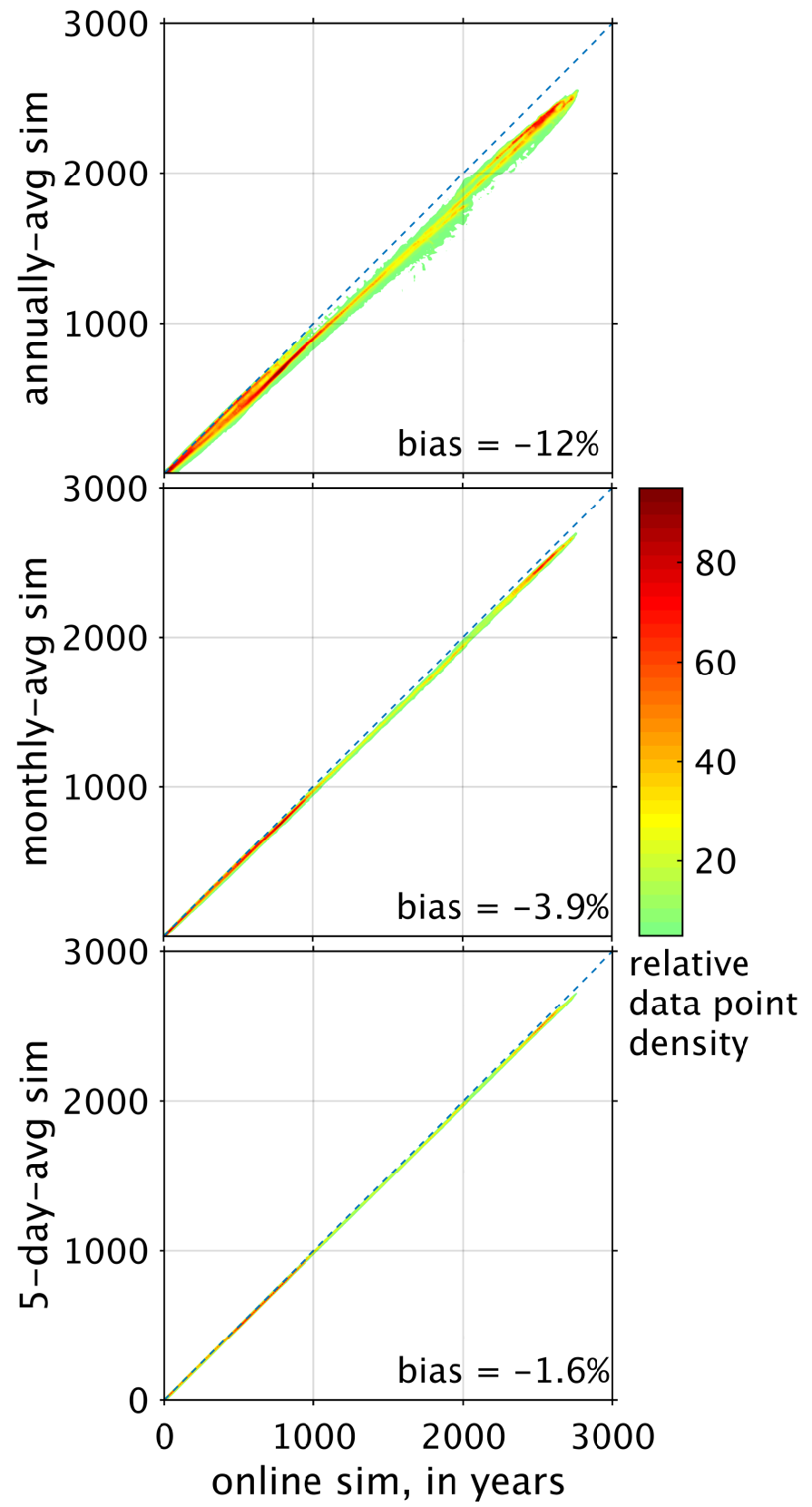

Figure 2: Scatter density diagrams comparing the equilibrium solutions for ideal age using annually-averaged (top), monthly-averaged (middle), and 5-day-averaged (bottom), transport matrices versus using an online simulation. The "bias" is the average percent difference, on a volume weighted basis, when using the transport matrices rather than using an online simulation. The ideal age is younger using the transport matrices than when using the online simulation. 


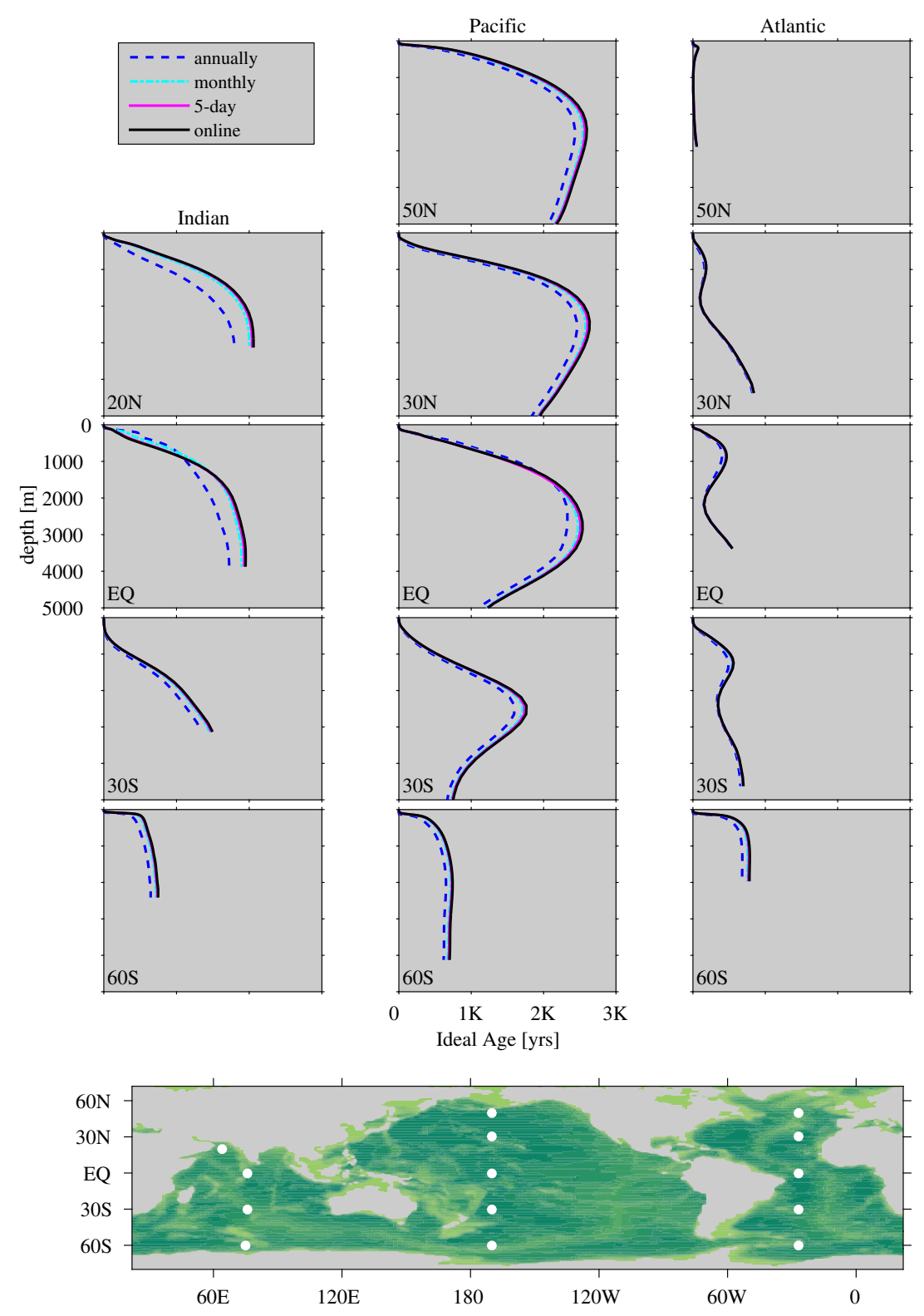

Figure 3: Depth profiles in meters of ideal age in years at selected locations in the Indian, Pacific, and Atlantic basins. The profiles compare the equilibrium solutions when the one-year simulation is run online versus using annually-averaged, monthly-averaged, and 5-day-averaged transport matrices offline. Only the profile using the annually-averaged transport matrices is clearly distinguishable from the online simulation. The topographical map shows the locations of the depth profiles. 
The scatter-density diagrams provide information on how the ideal age computed in each of the cases differs with age. To examine how each case varies with depth, profiles of ideal age versus depth are provided. Figure 3 shows depth profiles at locations throughout the ocean for the cases where the simulation used the transport matrices, and for the case where the online simulation was used. The accompanying topographical map shows the locations of the profiles. The right-hand column of profiles is in the Atlantic Basin, going from $50^{\circ} \mathrm{N}$ to $60^{\circ} \mathrm{S}$ traveling from top to bottom. The ideal age is nearly the same in all cases except for the profile in the Southern Ocean.

The Pacific basin, the middle column in Figure 3, shows a smaller but discernible difference for the annually-averaged transport matrix case, with the shapes of the profiles being similar. The largest differences are in the older waters, between 2000 and $4000 \mathrm{~m}$. At $3000 \mathrm{~m}$ near the equator, the online simulation results in an ideal age of 2538 years. The difference with the annually-averaged case is 220 years; with the monthly-averaged case it is 69 years; and with the 5 -day-averaged case it is 29 years; indicating relative errors of $9 \%, 3 \%$, and $1 \%$ respectively. If one considers a deep water parcel migrating slowly from the Southern Ocean northwards, and returning southwards at depths of around $3000 \mathrm{~m}$, that is consistent with the age profiles. It also is consistent with the absolute value of the bias becoming larger as the water increases in age; however, the relative (\%) bias does not.

The Indian Basin, shown in the left-hand column, has the largest difference between the annually-averaged transport matrices and all of the other cases. The northern Indian Ocean is an area of exceptionally strong seasonal variability. The largest differences are in the north end of the basin, in the 
Arabian Sea (the $20^{\circ} \mathrm{N}$ panel); this is consistent with a comparison between the annually-averaged and monthly-averaged cases in Bardin et al. (2014). While the online simulation results in an ideal age of 1870 years at $20^{\circ} \mathrm{N}$ and $2000 \mathrm{~m}$ depth, the annually-averaged case is 409 years younger, the monthlyaveraged case is 59 years younger, and the 5-day-averaged case is 20 years younger, corresponding to relative errors of $22 \%, 3 \%$, and $1 \%$ respectively. Near the equator at $2000 \mathrm{~m}$ depth, the online simulation results in an ideal age of 1705 years. The annually-averaged case is 297 years younger; the monthly-averaged case is 66 years younger; and the 5-day-averaged case is 19 years younger; indicating relative errors of $17 \%, 4 \%$, and $1 \%$ respectively. The three profiles in the Southern Ocean at $60^{\circ} \mathrm{S}$ are remarkably similar, within a few years of each other at the same depth. At $2000 \mathrm{~m}$, the online simulation results in an ideal age of around 750 years; the difference for the annually-averaged case is 101 years; the monthly-averaged case is 28 years younger; and the 5-day-averaged case 13 years younger; indicating relative errors of $13 \%, 4 \%$, and $2 \%$ respectively.

In Figures 4, 5, 6, and 7 we show the relative error for the ideal age computation based on the annually-averaged, monthly-averaged, and 5-day averaged transport operator at $400 \mathrm{~m}, 1000 \mathrm{~m}, 3000 \mathrm{~m}$, and $4000 \mathrm{~m}$ depths. For all cases the relative error decreases with increasing temporal resolution of the transport matrices.

At $400 \mathrm{~m}$ (Figure 4), a band near the equator as well as the Southern and South-Eastern coast of Australia show older ideal ages for the offline model, while the rest of the ocean, and especially the high-latitude North Atlantic Ocean and Eastern Mediterranean basin show a youthful bias. 

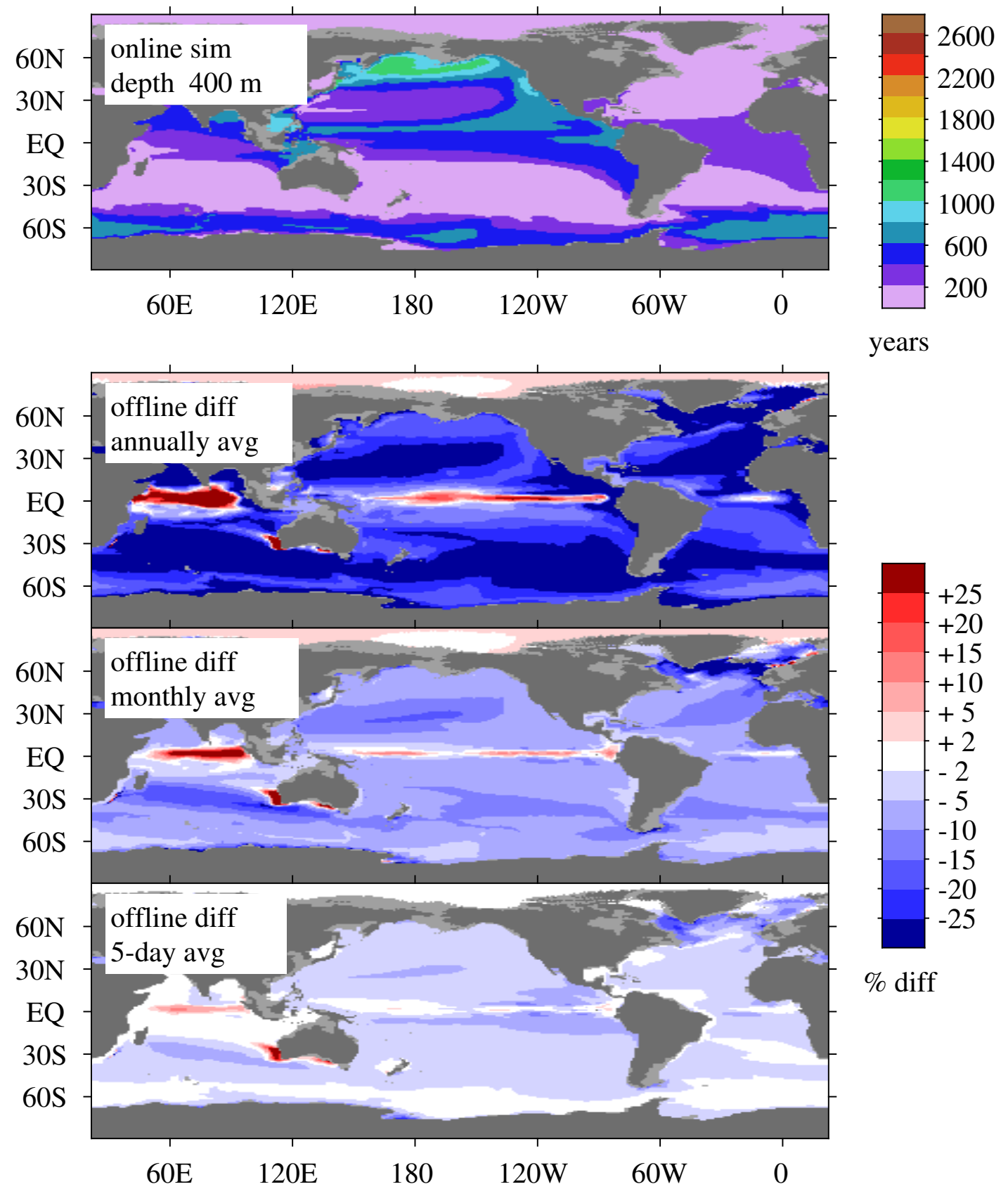

Figure 4: At a depth of $400 \mathrm{~m}$, the top panel shows the equilibrium ideal age using the online simulation. The bottom three panels show the percent difference when the simulation is run using the annually-averaged, monthly-averaged, and 5-day averaged transport matrices. A positive percent difference means the offline model has older ideal age than the online model. 

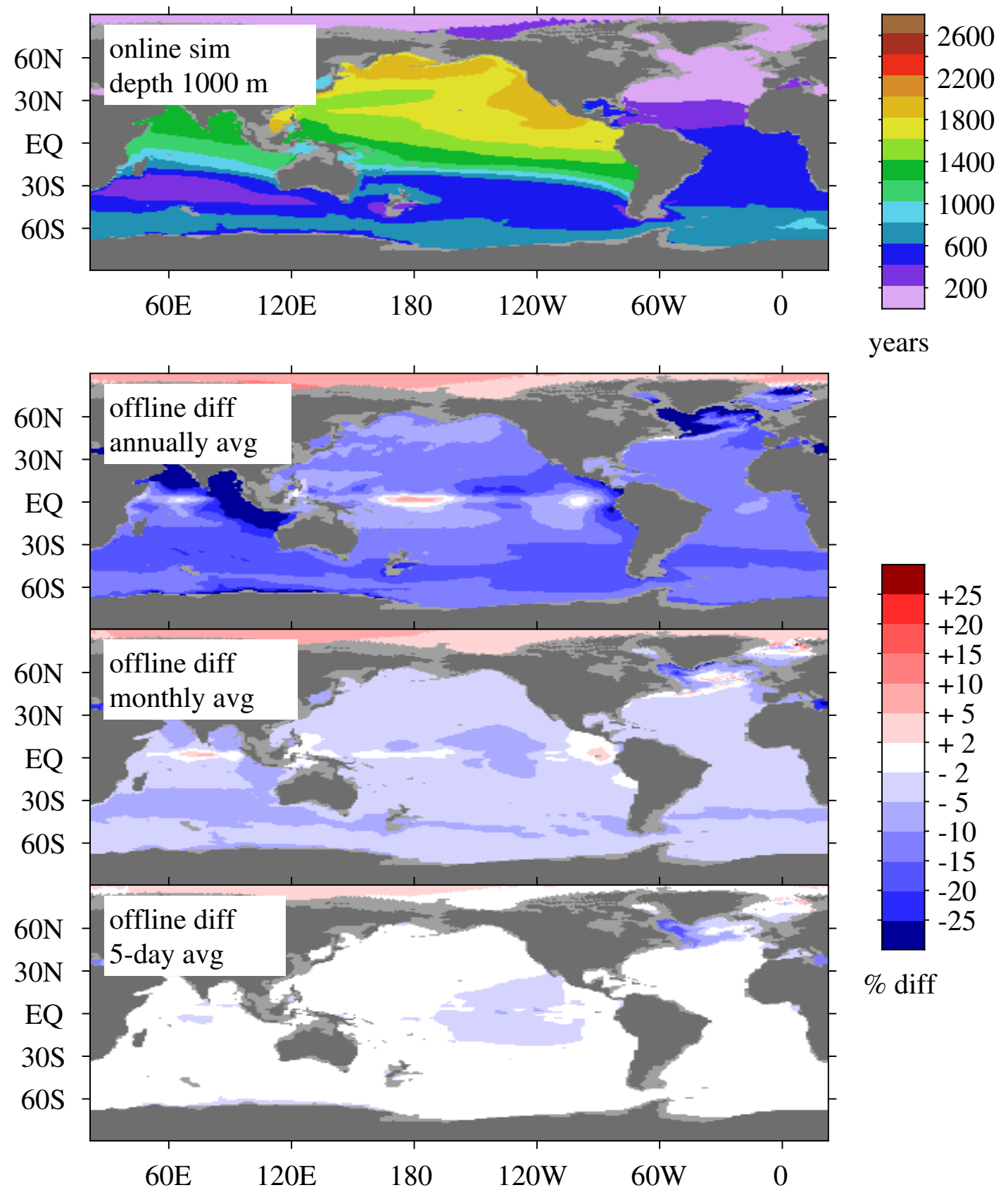

Figure 5: At a depth of $1000 \mathrm{~m}$, the top panel shows the equilibrium ideal age using the online simulation. The bottom three panels show the percent difference when the simulation is run using the annually-averaged, monthly-averaged, and 5-day averaged transport matrices. A positive percent difference means the offline model has older ideal age than the online model. 

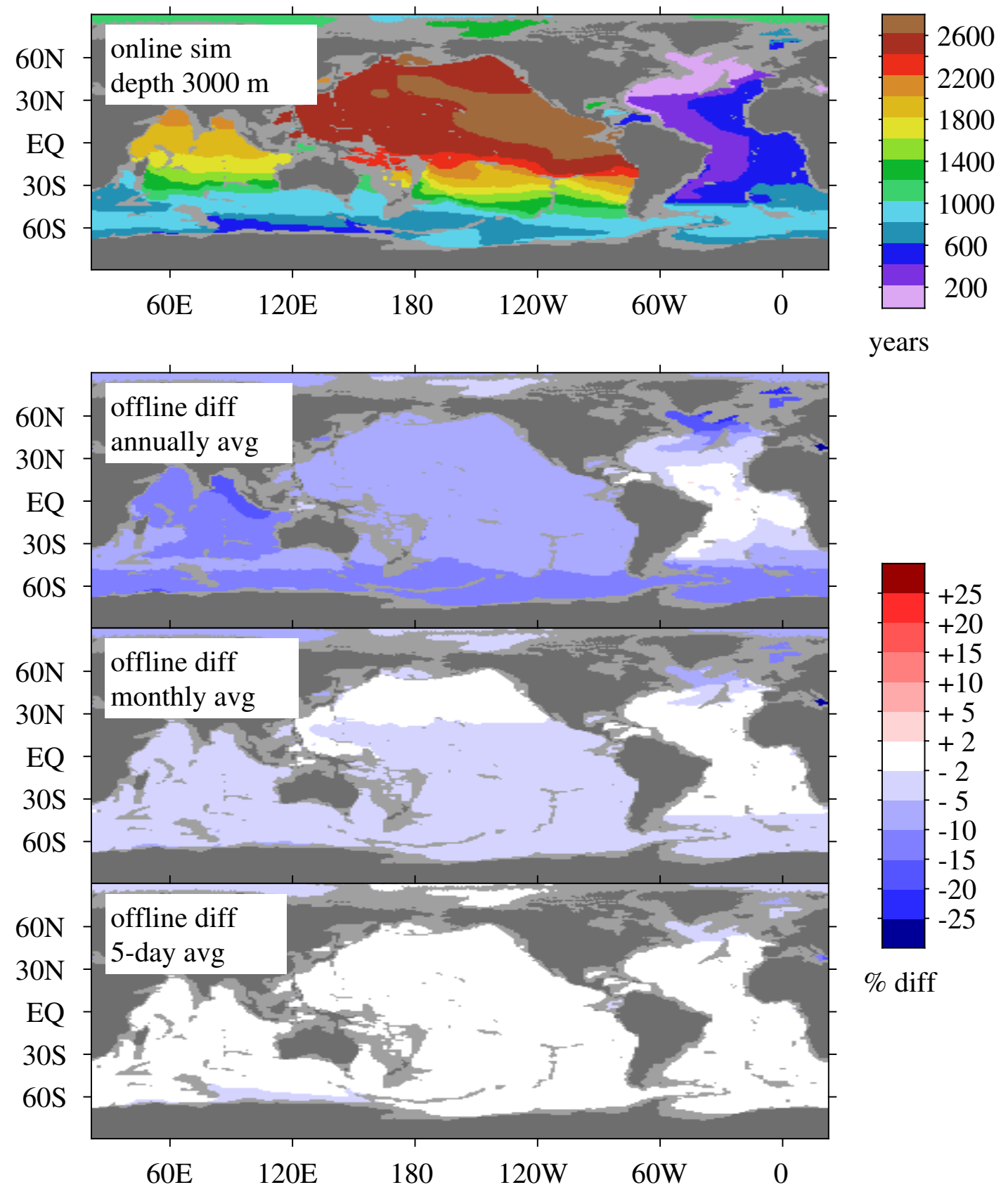

Figure 6: At a depth of $3000 \mathrm{~m}$, the top panel shows the equilibrium ideal age using the online simulation. The bottom three panels show the percent difference when the simulation is run using the annually-averaged, monthly-averaged, and 5-day averaged transport matrices. A positive percent difference means the offline model has older ideal age than the online model. 

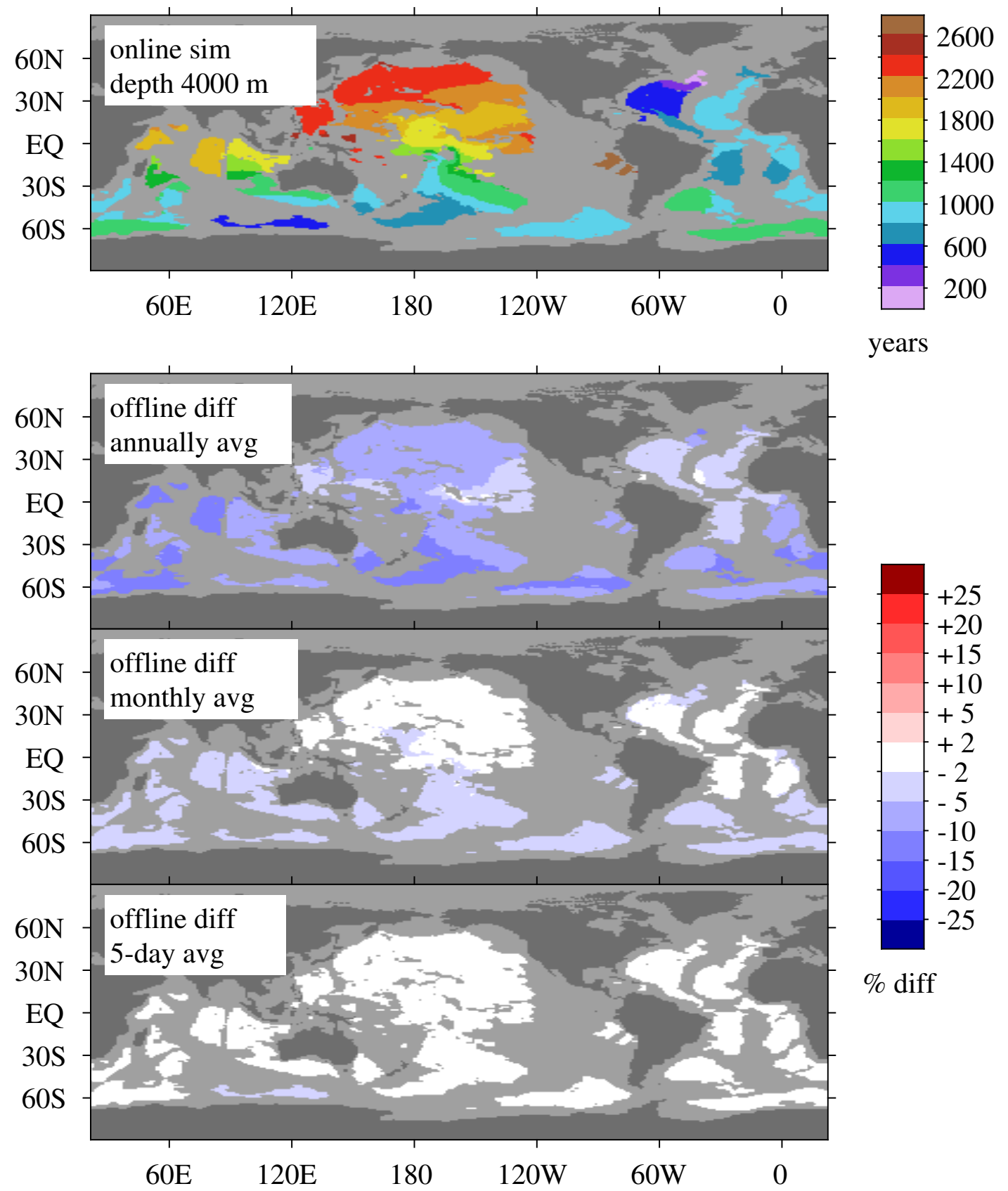

Figure 7: At a depth of $4000 \mathrm{~m}$, the top panel shows the equilibrium ideal age using the online simulation. The bottom three panels show the percent difference when the simulation is run using the annually-averaged, monthly-averaged, and 5-day averaged transport matrices. A positive percent difference means the offline model has older ideal age than the online model. 
At $1000 \mathrm{~m}$ (Figure 5), the relative differences with the online simulation are less than for the $400 \mathrm{~m}$ case. The Labrador Sea and the Mediterranean are still showing a substantial youthful bias. For the annually-averaged case, the percent differences in the North Pacific, where some of the oldest waters reside, are significantly less than the $400 \mathrm{~m}$ case.

At $3000 \mathrm{~m}$ (Figure 6), the age bias along the equator that was evident higher up in the water column disappears. The relative error field shows a youthful bias at high latitudes where deep waters are newly formed. In general this relative error decreases as water moves away from the high latitude formation regions, indicating that there is little accumulation of transport errors along the flow. A notable exception is the north-eastern Indian Ocean basin where masses have a local relative error maximum for the annuallyaveraged operator and for the monthly-averaged operators.

The ages at $4000 \mathrm{~m}$ (Figure 7), reflect the pattern seen at $3000 \mathrm{~m}$. The differences of the monthly-averaged case and the 5-day averaged case with the online simulation are relatively small.

\section{Discussion}

The general agreement between the online equilibrium solution and the solutions using the offline transport matrices gives confidence that the offline simulated equilibrium solution is representative of the online model behavior. For applications where the interest is in the ventilation of the deep ocean, even the annually-averaged transport operator captures the first order ventilation patterns of the parent model. For applications where relative errors of up to $10 \%$ are tolerable, the monthly averaged operators appear adequate 
for waters below $1000 \mathrm{~m}$; the 5-day averaged operators have relative errors that are generally less than $10 \%$ for depths extending up to $400 \mathrm{~m}$ below the surface. We note these errors are small compared to the errors of the online model in relation to observationally based ideal age estimates, which are on the order of $100 \%$ in the deep North Pacific basin, i.e. $\sim 2800$ years for CESM-POP2 versus $\sim 1400$ years for the observationally constrained model of DeVries and Primeau (2011).

For the upper-most ocean, the ideal age is not the best tracer with which to evaluate the offline tracer transport matrix. Because the ideal age is identically zero at the surface, it is not a good tracer to capture the strong seasonality of the circulation in the surface layers. Other tracers that have more realistic boundary conditions at the surface would provide a better means of evaluating the temporal resolution of the tracer transport matrices in the upper-most ocean.

Given that the offline matrix approach results in some bias with respect to the online model (unless the transport matrices are saved for every timestep of the parent model), one might wonder what the advantages of using the offline approach are that make it compelling. The main reasons are the ease with which new tracers can be implemented in the offline model and the substantial savings in computer resources; these lead to a much shorter real time to generate a close approximation. The savings in computer resources can be illustrated by those used in the one-year simulation: for similar wallclock times, the online simulation uses 16 computer nodes, compared to 1 computer node when using the monthly-averaged transport matrices. In the offline model, tracer source-sink functions can be easily coded in a high 
level language like Matlab without much of a performance penalty because most of the heavy computational lifting consists of matrix-vector products and the solution of a tri-diagonal system of linear equations. While these heavy computational operations can easily be implemented in Matlab with a single line of code, we have found it beneficial to implement them in $\mathrm{C}++$ to reduce the computational time; however, a modeler need not be aware of these details to implement new tracer source-sink functions in the offline model. Appendix A provides software design details. Appendix B provides more computer resource details and a table of comparisons for the online and various offline simulations.

\section{Conclusions}

We have used the implicit Newton-Krylov method to obtain the equilibrium solution for ideal age using the offline annually-averaged, monthlyaveraged, and 5-day-averaged transport matrices, and using the online dynamic model itself. This has shown that compared to using the online dynamic model, a volume-weighted bias of $12 \%$ younger results when annuallyaveraged transport matrices are used, but only $3.9 \%$ younger when monthlyaveraged transport matrices were used, and $1.6 \%$ younger when 5-day averaged transport matrices were used. That these errors decrease as the temporal resolution of the transport matrix operator increases confirms that the transport matrices faithfully capture the circulation of the parent model. Furthermore, the relative errors incurred when computing the ventilation ages of the deep ocean using monthly-averaged transport matrices are small relative to the age biases of the online model in relation to the observations. 
This makes the offline transport matrix model a reasonably accurate method that is easy-to-use and computationally efficient for simulating tracer distributions in equilibrium with their source-sink functions.

\section{Acknowledgments}

This work was supported by the Department of Energy, Office of Science, Biological and Environmental Research, under SciDAC award number DE-SC0012550 at the University of California, Irvine, and under DOE Field Work Proposal 2011 SNL 014938 at Sandia National Laboratories. Sandia National Laboratories is a multiprogram laboratory managed and operated by Sandia Corporation, a wholly owned subsidiary of Lockheed Martin Corporation, for the U. S. Department of Energy's National Nuclear Security Administration under Contract No. DE-AC04-94AL85000. Computing resources were provided by the Climate Simulation Laboratory at the National Center for Atmospheric Research's Computational and Information Systems Laboratory, sponsored by the National Science Foundation and other agencies. We thank Weiwei Fu for helpful discussions and support in validating the transport matrix build procedures. We thank two anonymous reviewers for their constructive comments.

\section{Appendix A. Computation Details}

$\mathrm{C}++$ coding was used to enhance the performance of the matrix-vector products and the solution of the tri-diagonal system of linear equations. These occur during the one-year simulation, which uses the bulk of the computer time. The $\mathrm{C}++$ Matlab "mex" interface function implements three 
OpenMP-multithreaded computational kernels: the matrix-vector product, the tri-diagonal matrix factorization, and the subsequent linear equation solution. The tri-diagonal matrix routines exploit the property that the vertical diffusion operation is decoupled by physical water column. Hence each thread can factor the part of a matrix associated with just one water column, and similarly can solve just the associated part of the linear equation. Each such block is factored using Lapack's "dgttrf" function, and the solver uses the "dgttrs" function (Anderson et al., 1999).

\section{Appendix B. Computer Resources}

A comparison of the computer resources and wall-clock time for running one-year tracer simulations using the online and offline model is summarized in Table B.1. On the order of 20-30 one-year simulations are needed for the Newton-Krylov solver to converge. The comparisons are from runs accomplished at the NCAR Yellowstone facility. There are several types of processors at the facility. The "Yellowstone" processors are 2.6-GHz Intel Xeon ES-2670 processors; each "Yellowstone" node has 16 of these processors, and 32 GB of memory. "Geyser" processors are 2.4-GHz Intel Xeon E7-4870 processors; each "Geyser" node has 40 processors and 1 TB of memory.

The table also shows the computational time for obtaining the equilibrium ideal age for the annually averaged flow by direct matrix inversion using the MUMPS solver (Amestoy et al., 2001). This is feasible because the annually-averaged transport matrix is a steady-state transport operator. A direct solution is provided for $\mathbf{T} a=S$, where $S$ is a constant source-sink term. The total wall-clock time for the direct solution of the steady-state 

configuration because of the large memory availability.

\begin{tabular}{|l||l|l|}
\hline Simulation Type & Nodes & Wall-Clock Time \\
\hline \hline online & 16 Yellowstone & 27 min. per model year \\
\hline annually-averaged & 1 Yellowstone & 19 min. per model year \\
\hline monthly-averaged & 1 Yellowstone & 19 min. per model year \\
\hline 5-day-averaged & 1 Yellowstone & 49 min. per model year \\
\hline annually-averaged (MUMPS) & 1 Geyser & 30 min. equilibrium solution \\
\hline \hline
\end{tabular}

Table B.1: Wall-clock time and number of computer nodes used for online and offline one-year simulations at the NCAR Yellowstone facility. Each Yellowstone node has 16 processors and 32 GB of memory; each Geyser node has 40 processors and 1 TB of memory. Each Newton-Krylov solution takes 20 - 30 one-year simulations to converge to the equilibrium solution. MUMPS uses a direct matrix inversion to compute the equilibrium solution. minutes versus 9 hours); but the memory requirement is much larger (300 GB versus 112 GB using Newton-Krylov on a time-stepping simulation with an in-memory factored preconditioner, or 6 GB for the time-stepping simulation only). The direct solution is most efficiently executed on the "Geyser" (1)

\section{References}

Amestoy, P.R., Duff, I.S., Koster, J., L'Excellent, J.Y., 2001. A fully asynchronous multifrontal solver using distributed dynamic scheduling. SIAM Journal of Matrix Analysis and Applications 23, 15-41.

Anderson, E., Bai, Z., Bischof, C., Blackford, S., Demmel, J., Dongarra, J., DuCroz, J., Greenbaum, A., Hammarling, S., McKenney, A., Sorensen,

transport model is significantly less than when Newton-Krylov is used (30 
D., 1999. \{LAPACK\} Users' Guide. Society for Industrial and Applied Mathematics, Philadelphia, PA. third edition.

Bardin, A., Primeau, F., Lindsay, K., 2014. An Offline Implicit Solver for Simulating Prebomb Radiocarbon. Ocean Modelling 73, 45-58.

Briegleb, B.P., Danabasoglu, G., Large, W.G., 2010. An Overflow Parameterization for the Ocean Component of the Community Climate System Model. NCAR Technical Note. NCAR.

Danabasoglu, G., Large, W.G., Briegleb, B.P., 2010. Climate Impacts of Parameterized Nordic Seas Overflows. Journal of Geophysical Research Oceans submitted.

Deleersnijder, E., Campin, J.M., Delhez, E.J.M., 2001. The concept of age in marine modelling I. Theory and preliminary model results. Journal of Marine Systems 28, 229-267.

DeVries, T., Primeau, F., 2011. Dynamically- and observationallyconstrained estimates of water-mass distributions and ages in the global ocean. Journal of Physical Oceanography 41, 2381-2401.

England, M.H., 1995. The Age of Water and Ventilation Timescales in a Global Ocean Model. Journal of Physical Oceanography 25, 2756-2777.

Gent, P.R., McWilliams, J.C., 1990. Isopycnal Mixing in Ocean Circulation Models. Journal of Physical Oceanography 20, 150-155.

Graven, H.D., Gruber, N., Key, R., Khatiwala, S., Giraud, X., 2012. Changing controls on oceanic radiocarbon: New insights on shallow-to-deep ocean 
exchange and anthropogenic CO2 uptake. Journal of Geophysical Research 117, C10005.

Griffies, S.M., Biastoch, A., Boning, C., Bryan, F., Danabasoglu, G., Chassignet, E.P., England, M.H., Gerdes, R., Haak, H., Hallberg, R.W., Hazeleger, W., Jungclaus, J., Large, W.G., Madec, G., Pirani, A., Samuels, B.L., Scheinert, M., Sen Gupta, A., Severijns, C.A., Simmons, H.L., Trequier, A.M., Winton, M., Yeager, S., Yin, J.J., 2009. Coordinated ocean-ice reference experiments (COREs). Ocean Modelling 26, 1-46.

Khatiwala, S., 2008. Fast spin up of Ocean biogeochemical models using matrix-free Newton-Krylov. Ocean Modelling 23, 121-129.

Khatiwala, S., Visbeck, M., Cane, M.A., 2005. Accelerated simulation of passive tracers in ocean circulation models. Ocean Modelling 9, 51- 69.

Kriest, I., Oschlies, A., 2011. Numerical effects on organic-matter sedimentation and remineralization in biogeochemical ocean models. Ocean Modelling 39, 275-283.

Kwon, E.Y., Primeau, F., 2006. Optimization and sensitivity study of a biogeochemistry ocean model using an implicit solver and in situ phosphate data. Global Biogeochemical Cycles 20.

Large, W.G., McWilliams, J.C., Gent, P.R., Bryan, F.O., 1994. Oceanic vertical mixing: A review and a model with a nonlocal boundary layer parameterization. Reviews of Geophysics 32, 363-403.

Large, W.G., Yeager, S.G., 2004. Diurnal to decadal global forcing for ocean 
and sea-ice models: The data sets and flux climatologies. NCAR Technical Report TN-460+STR.

Large, W.G., Yeager, S.G., 2009. The global climatology of an interannually varying air-sea flux data set. Climate Dynamics 33, 341-364.

Letscher, R.T., Moore, J.K., Teng, Y.C., Primeau, F., 2015. Variable C : N : P stoichiometry of dissolved organic matter cycling in the Community Earth System Model. Biogeosciences 12, 209-221.

Li, X., Primeau, F., 2008. A fast Newton-Krylov solver for seasonally varying global ocean biogeochemistry models. Ocean Modelling 23, 13-20.

Lindsay, K., submitted. A Newton-Krylov solver for fast spin-up of online ocean tracers. Ocean Modelling .

Prieß, M., Piwonski, J., Koziel, S., Oschlies, A., Slawig, T., 2013. Accelerated parameter identification in a 3D marine biogeochemical model using surrogate-based optimization. Ocean Modelling 68, 22-36.

Primeau, F., 2005. Characterizing Transport between the Surface Mixed Layer and the Ocean Interior with a Forward and Adjoint Global Ocean Transport Model. Journal of Physical Oceanography 35, 545-564.

Primeau, F., Deleersnijder, E., 2009. On the time to tracer equilibrium in the global ocean. Ocean Science 5, 13-28.

Redi, M.H., 1982. Oceanic Isopycnal Mixing by Coordinate Rotation. Journal of Physical Oceanography 12, 1154-1158. 
490 Smith, R., Jones, P., Briegleb, B., Bryan, F., Danabasoglu, G., Dennis, J., ${ }_{491}$ Dukowitz, J., Eden, C., Fox-Kemper, B., Gent, P., Hecht, M., Jayne, S., ${ }_{492}$ Jochum, M., Large, W., Lindsay, K., Maltrud, M., Norton, N., Peacock, ${ }_{493}$ S., Vertenstein, M., Yeager, S., 2010. Parallel Ocean Program (POP) 494 Reference Manual. 